\title{
Immunohistochemical Nucleocytoplasmic Localization of Light Chain3 in the Keratinocytes of Psoriatic Skin Rehab Monir Samaka ${ }^{1}$, Alaa Hasan Marae ${ }^{2}$, Manar Ali Faried ${ }^{* 3}$
}

Departments of ${ }^{1}$ Pathology and ${ }^{2}$ Dermatology, Andrology \& STDs, Faculty of Medicine, Menoufia University, Menoufia, Egypt, ${ }^{3}$ Dermatologists, Menoufia Governorate, Egypt

*Corresponding author: Manar Ali Faried, Mobile: (+20) 01281060901, E-Mail: manarfaried@ yahoo.com

\begin{abstract}
Background: Light chain 3 is a sensitive autophagy-related protein distributed within the mammalian tissues. The role of LC3 localization in psoriasis pathogenesis is still poorly understood.

Objective: This study aimed to investigate, for the first time up to our knowledge, the localization of Light chain3 (LC3) in the keratinocytes of psoriatic skin by immunohistochemical study.

Materials and methods: This prospective case case-control study was carried out on 30 patients presented with chronic plaque psoriasis versus 30 age and gender-matched apparently healthy volunteers. Clinical data were collected and Psoriasis Area and Severity Index (PASI) was assessed. From all controls and cases (lesional and perilesional), skin biopsies were taken and the epidermis was assessed for histopathological changes and LC3 immunoreaction. Results: There was a highly significant difference $(\mathrm{P}<0.001)$ between the control skin and psoriatic skin (lesional and perilesional) regarding the epidermal LC3 localization. Nucleocytoplasmic LC3 localization was dominant in lesional skin specimens. Conclusion: Nucleocytoplasmic localization of LC3 in the keratinocytes of the psoriatic skin might play a pivotal role in psoriasis pathogenesis. This can open a new gate for target therapy in psoriasis.
\end{abstract}

Keywords: Psoriasis, Autophagy, LC3, Immunohistochemistry.

\section{INTRODUCTION}

Psoriasis is a chronic, inflammatory immunemediated skin disease characterized by epidermal hyperplasia, defective keratinization, and infiltration of immune cells within the skin ${ }^{(1)}$. Psoriasis prevalence is now in increase. It is a serious dermatological disease extending beyond the physical symptoms as it exerts a significant, negative impact on patients' quality of life ${ }^{(2)}$.

The pathogenesis of psoriasis is not completely understood ${ }^{(3)}$. Autophagy, an intracellular catabolic process associated with the maintenance of cellular homeostasis, plays a critical role in inflammation, autoimmunity, and cellular differentiation ${ }^{(4)}$.

Many autophagy-related proteins (Atgs) have been identified in autophagosome formation. Some of the Atgs such as beclin 1, p62, and light chain 3 (LC3) are more sensitive and stable during autophagosome formation and therefore can act as markers for autophagosomes ${ }^{(\mathbf{5})}$. Microtubule-associated protein LC3 is a soluble protein with a molecular mass of approximately $17 \mathrm{kDa}$ that is distributed ubiquitously in mammalian tissues and cultured cells ${ }^{(6)}$. The exact localization of LC3 is essential for investigating the role of autophagy in psoriasis. Therefore, this study aimed to assess the nucleocytoplasmic localization of LC3 within the keratinocytes of the psoriatic skin.

\section{PATIENTS AND METHODS}

This prospective case-control study was carried out on a total number of 60 subjects, they included 30 patients with chronic plaque psoriasis and 30 age and gender-matched apparently normal subjects as a control group. Patients were selected randomly from Dermatology Outpatient Clinic, Menoufia University
Hospital in the period between December 2018 and December 2019. Control subjects were selected from apparently healthy persons attending the Plastic Surgery Department, Menoufia University Hospital.

\section{Ethical Considerations}

A written consent form, approved by the Local Ethical Research Committee in the Faculty of Medicine, Menoufia University, was obtained from every participant before study initiation.

Exclusion criteria include: a) Cases of other types of psoriasis b) Treated patients with any topical or systemic therapy for at least four months before the study c) Any dermatological diseases except chronic plaque psoriasis d) Any associated inflammatory or autoimmune disease e) Refusal of patients.

Every patient was subjected to complete history taking and physical examination. The data collected included the age, gender, onset, course, duration of the disease, site of affection, and presence of itching. Full general and dermatological examinations were done. The severity of the disease was assessed by the Psoriasis Area and Severity Index (PASI) score ${ }^{(7)}$. Mild psoriasis means a PASI score of $\leq 10$ while moderate to severe psoriasis PASI score of $>10^{(8)}$.

\section{Skin biopsies:}

Skin biopsies were taken under $2 \%$ lignocaine local anesthesia. For each patient, a skin biopsy from lesional skin and another biopsy from perilesional skin was taken. The biopsies from the lesional skin were taken within the lesion, $1 \mathrm{~cm}$ from the edge of the plaque border ${ }^{(9)}$, whereas the biopsies from the perilesional skin were taken $2 \mathrm{~cm}$ beyond the plaque border ${ }^{(\mathbf{1 0})}$. Skin biopsies 
were taken with a $3 \mathrm{~mm}$ punch from each patient and the apparently healthy normal controls.

All biopsies were submitted to the Pathology Department, Faculty of Medicine, Menoufia University. They were fixed in $10 \%$ neutral buffered formalin, dehydrated in ascending grades of ethanol followed by immersion in xylene then impregnated in paraffin. Several 5 micron thick sections from each block were taken. One slide was stained by Hematoxylin and Eosin for routine histopathological examination. Other sections were mounted on positively charged slides and stored at room temperature for immunohistochemical staining for the LC3 antibody.

\section{Pathological parameters of the studied psoriasis group:}

Epidermal histopathological changes in the lesional and perilesional skin specimens were examined and the different degrees for each parameter were assessed subjectively. Epidermal changes included acanthosis, psoriasiform hyperplasia, hyperkeratosis, parakeratosis, Munro's microabscess, a spongiform pustule of Kogoj, granular cell layer status, suprapapillary thinning, spongiosis, tips of rete ridges, and exocytosis.

\section{Immunohistochemical staining for LC3:}

Immunohistochemical staining was performed using Rabbit Polyclonal Anti- LC3 antibody (Cat. No. YPA1340). Chongqing Biospes Co., Ltd (Room 272, Building 7, No.15, Paradise Walk, Jiangbei District, Chongqing, 400020, China). It was received in a single vial containing $1 \mathrm{ml}$ of concentrated antibody.

Immunohistochemical staining was performed using the Universal Dako cytomation Labelled Streptavidin-Biotin-2 system. The primary antibody (LC3, 1:150) was applied on the slides and incubated overnight at room temperature in a humidity chamber. Finally, the detection of bound antibody was accomplished using a modified labeled avidin-biotin (LAB) reagent for 20 minutes then phosphate Buffered Saline (PBS) wash. A $0.1 \%$ solution of diaminobenzidine (DAB) was used for 5 minutes as a chromogen. Slides were counterstained with Mayer's hematoxylin for 5-10 minutes.

The status of positivity and the pattern of expression of the epidermal LC3 immunoreaction for the lesional and perilesional stained slides for each patient as well as the normal individual were assessed.

\section{Statistical Analysis}

Data were collected, tabulated, and analyzed by statistical package for social science (SPSS) version 25 on IBM compatible computer (SPSS Inc., Chicago, IL, USA). Chi-square and/or Fisher exact test and One-way ANOVA were used. P-value $\leq 0.05$ was considered statistically significant and $\mathrm{P}$-value $<0.001$ was considered highly significant.

\section{RESULTS}

\section{Clinical data of the psoriasis group:}

The onset of the disease was early in $19(63.3 \%)$ cases and late in $11(36.7 \%)$ cases. The course of the disease was stationary in $16(53.3 \%)$ cases and progressive in $14(46.7 \%)$ cases. The duration of the disease ranged from 1-33 years. The trunk was affected in $1(3.3 \%)$ case, extremities were affected in $5(16.7 \%)$ cases, while both trunk and extremities were affected in $24(80 \%)$ cases. The scalp was affected in $13(43.3 \%)$ cases and wasn't affected in $17(56.7 \%)$ cases. Palms and soles were affected in $2(6.7 \%)$ cases and weren't affected in $28(93.3 \%)$ cases. Nail affection was present in 19 $(63.3 \%)$ cases and absent in 11 (36.7) cases. Koebnerization was present in $10(33.3 \%)$ and absent in $20(66.7 \%)$ cases. The itching was complained by 17 $(56.7 \%)$ cases.

Ten $(33.3 \%)$ cases were smokers. Family history was positive in $11(36.7 \%)$ cases and negative in 19 (63.3\%) cases. Moreover, the PASI score ranged from 235 with mean \pm SD $13.23 \pm 7.88$. According to the PASI score, $16(53.3 \%)$ cases were with mild psoriasis while $14(46.7 \%)$ cases were with moderate to severe psoriasis.

\section{Histopathological data:}

1. Comparison between the lesional and perilesional skin of the psoriasis group regarding the epidermal histopathological parameters (Table 1):

There were highly significant differences between lesional and perilesional skin regarding the presence of acanthosis and its degree $(\mathrm{P}<0.001$ for both). There were highly significant differences between lesional and perilesional skin regarding the presence of psoriasiform hyperplasia and its degree $(\mathrm{P}<0.001$ and 0.022) respectively. There were highly significant differences between lesional and perilesional skin regarding the presence of hyperkeratosis and its degree ( $\mathrm{P}<0.001$ and 0.011 respectively). Moreover, there were highly significant differences between lesional and perilesional skin regarding the presence of parakeratosis $(\mathrm{P}=0.006)$ with no significant difference regarding its degree $(\mathrm{P}=1.0)$. There was a non-significant difference between lesional and perilesional skin regarding the presence of Munro microabscesses $(\mathrm{P}=0.49)$. Also, there was a highly significant difference between lesional and perilesional skin regarding the presence of suprapapillary thinning $(\mathrm{P}<0.001)$. A highly significant difference between lesional and perilesional skin regarding the granular cell layer $(\mathrm{P}<0.001)$ was recorded.

There was a significant difference between lesional and perilesional skin regarding the presence of spongiosis $(\mathrm{P}<0.001)$ and a non-significant difference regarding its degree $(\mathrm{P}=0.149)$. Also, there was a highly significant difference between lesional and perilesional skin regarding the morphology of rete ridges' tips $(\mathrm{P}<0.001)$. There was a highly significant difference between lesional and perilesional skin regarding the presence of exocytosis $(\mathrm{P}=0.010)$ and a significant difference regarding its type $(\mathrm{P}=0.013)$ with no significant difference regarding its degree $(\mathrm{P}=0.092)$. 
Table (1): Comparison between lesional and perilesional skin of the psoriasis group regarding epidermal histopathological parameters

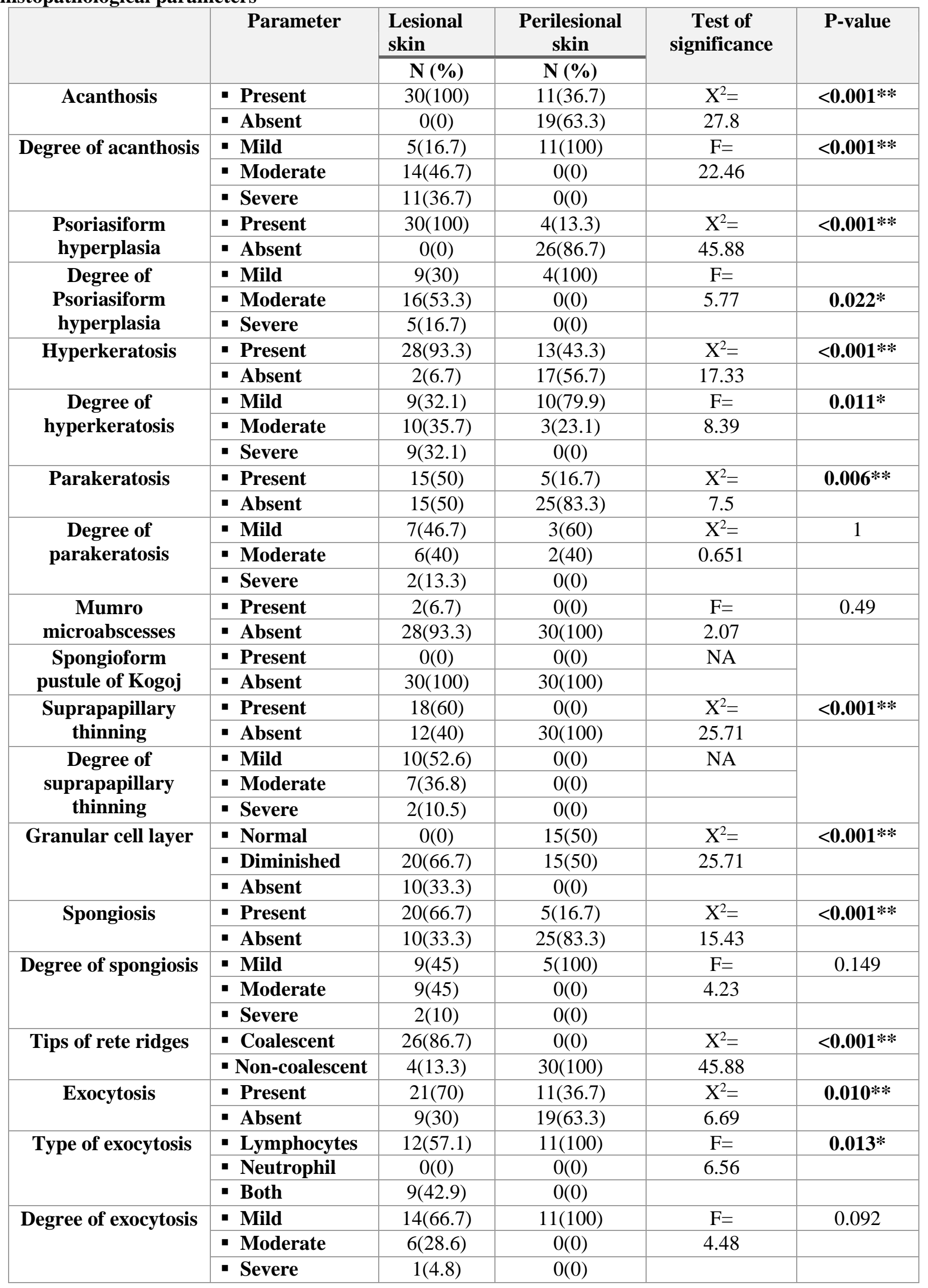


N: Number, $\mathbf{X}^{\mathbf{2}}$ : Chi=square test, F: Fisher's Exact test, NA: Non-applicable, *: Significant, **: Highly significant.

2. The immunohistochemical reaction of LC3 in control group and psoriasis group (lesional and perilesional skin):

In control skin, epidermal LC3 immunoreaction was positive in 29 (96.7\%) cases and negative in 1(3.3\%) case. Out of the positive cases, $14(48.3 \%)$ cases showed cytoplasmic expression, and $15(51.7 \%)$ cases displayed nucleocytoplasmic pattern. In perilesional skin, positive epidermal LC3 expression was noted in all examined samples. Most of them, 28 (93.3\%) cases, were with a nucleocytoplasmic pattern. Moreover, positive epidermal LC3 immunoreaction was observed in all examined samples of lesional skin. All the cases possessed nucleocytoplasmic LC3 immunoreaction (Figure 1, Table 2).
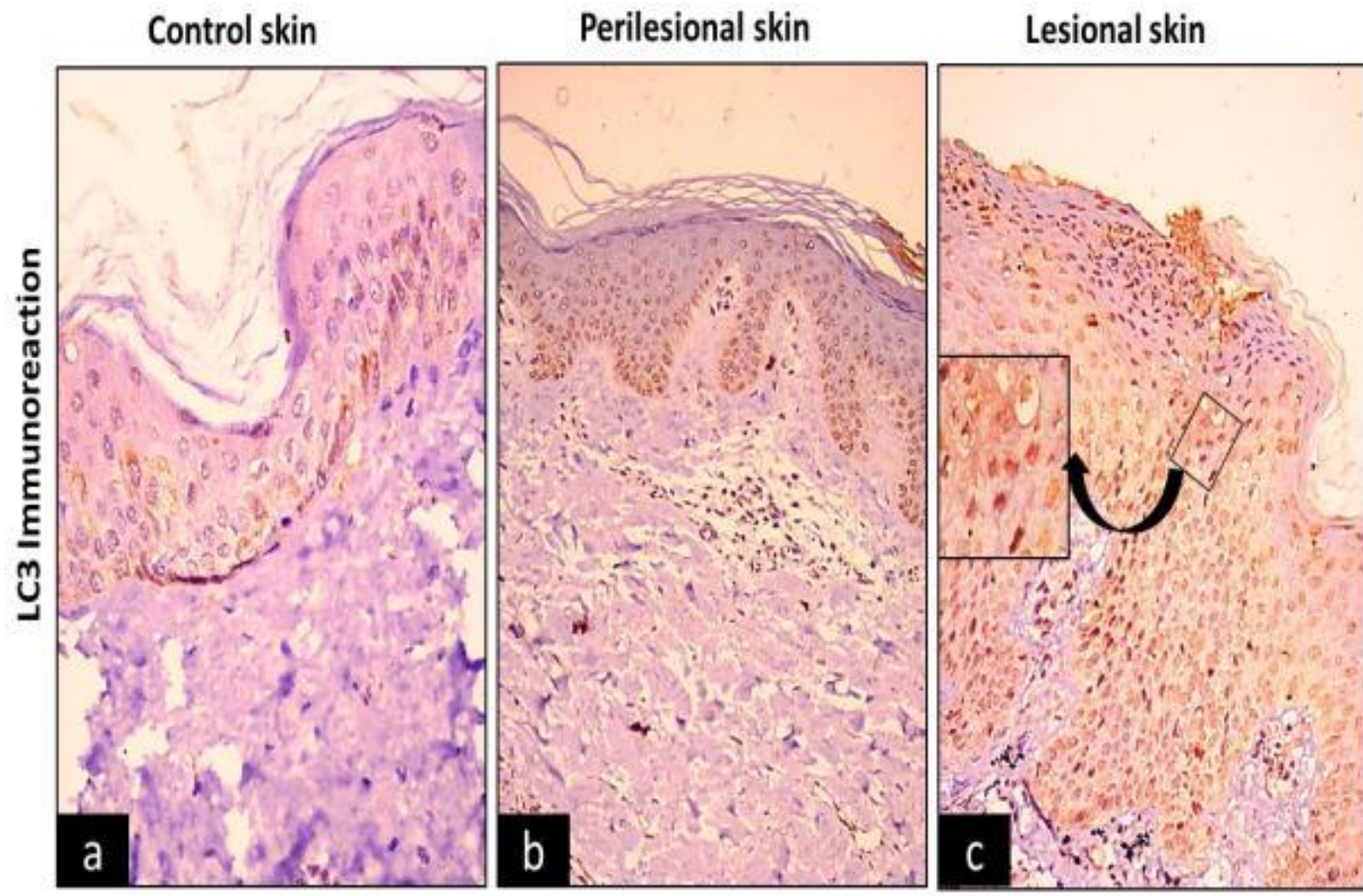

Figure (1): Photomicrographs of LC3 immunoreaction of the different studied groups. a) Control, b) Perilesional, c) Lesional. (LC3 x 200, inset x 400)

Table (2): Comparison between lesional and perilesional skin of the psoriasis group as well as the control group regarding the epidermal expression of LC3.

\begin{tabular}{|c|c|c|c|c|c|c|c|}
\hline Parameters & Lesional & Perilesional & Control & Test & P-value & $\begin{array}{c}\text { Test of } \\
\text { significance }\end{array}$ & P-value \\
\hline 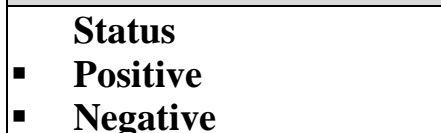 & $\begin{array}{c}\mathrm{N}(\%) \\
30(100) \\
0(0)\end{array}$ & $\begin{array}{c}\mathrm{N}(\%) \\
30(100) \\
0(0)\end{array}$ & $\begin{array}{c}\mathrm{N}(\%) \\
29(96.7) \\
1(3.3)\end{array}$ & $\begin{array}{c}\mathrm{F}= \\
1.84\end{array}$ & 1.0 & $\begin{array}{l}\text { NA } \\
1.017 \\
1.017\end{array}$ & $\begin{array}{c}\mathrm{b}=\mathbf{0 . 3 1 3} \\
\mathrm{c}=\mathbf{0 . 3 1 3}\end{array}$ \\
\hline $\begin{array}{l}\text { Pattern of expression } \\
\text { Cytoplasmic } \\
\text { Nucleocytoplasmic }\end{array}$ & $\begin{array}{c}0(0) \\
30(100)\end{array}$ & $\begin{array}{c}2(6.7) \\
28(93.3)\end{array}$ & $\begin{array}{l}14(48.3) \\
15(51.7)\end{array}$ & $\begin{array}{c}X^{2}= \\
27.23\end{array}$ & $<0.001$ & $\begin{array}{c}2.069 \\
12.917 \\
18.989\end{array}$ & $\begin{array}{c}\mathrm{a}=0.150 \\
\mathbf{b}<\mathbf{0 . 0 0 1} * * \\
\mathbf{c}<\mathbf{0 . 0 0 1} * *\end{array}$ \\
\hline
\end{tabular}

N: Number, $\mathbf{X}^{2}$ : Chi=square test, $\mathbf{F}$ : Fisher's Exact test **: Highly significant.

$\mathrm{a}=$ lesional vs perilesional $\quad \mathrm{b}=$ perilesional vs control $\quad \mathrm{c}=$ lesional vs control 


\section{DISCUSSION}

Psoriasis is a worldwide disease. It is a chronic recurrent, immune-mediated inflammatory skin disease. Unfortunately, psoriasis exerts unfavorable physical, emotional, and psychosocial effects on the affected patients ${ }^{(11)}$. The etiology of psoriasis is multifactorial. Recently, research on psoriasis pathogenesis has increased in a trial to get targeted and highly effective therapies ${ }^{(\mathbf{1 2})}$.

Autophagy has recently become a topic of great interest. It plays a pivotal role in maintaining cellular homeostasis by removing the damaged organelles and proteins. So, it is considered as a surviving mechanism ${ }^{(13)}$. In recent years, studies have shown that autophagy is a vital arbiter of cell fate decisions and plays an important role in inflammation, pathogen clearance, and antigen presentation ${ }^{(14)}$. The exact mechanism by which autophagy influences psoriasis is not well understood ${ }^{(15)}$. This work investigated, for the first time up to our knowledge, the localization LC3 in the keratinocyte of psoriasis patients as well as control subjects.

Recently, nucleophagy is a great topic of interest in many human diseases. Nucleophagy is selective autophagy that eliminates damaged nuclear material from a cell by autophagy pathway (16). Despite that nuclear LC3 plays crucial roles in autophagy, the mechanisms by which LC3 is targeted to the nucleus and nuclear components are poorly understood. It is clarified that LC3's trafficking into and out of the nucleus occurs in an organized manner and that the functions of nuclear LC3 are highly important ${ }^{(17)}$.

The present study seemed to be the first one, to the best of our knowledge, investigating the immunohistochemical LC3 localization is psoriatic skin. This study revealed that the nucleocytoplasmic localization of LC3 was significantly prominent in lesional psoriatic skin. This indicated the role of nucleophagy in psoriasis pathogenesis. This was following Shim et al. (18) who noted nuclear accumulation, via passive diffusion, of LC3 in response to cyclic mechanical stress and implied a surveillance role of nuclear LC3 against stretchinduced damage.

Although LC3 functions primarily in the cytoplasm where the autophagosomes and autolysosomes arise, LC3 is abundant in the nucleus ${ }^{(19)}$. Huang et al. ${ }^{(20)}$ considered the nuclear-derived pool of LC3 as the major source of autophagosometargeted LC3 in starved cells. Moreover, Huang and Liu ${ }^{(19)}$ considered the nuclear pool of LC3 as the primary source of membrane-conjugated LC3. They implicated the nucleocytoplasmic translocation of LC3 in priming starved cells in autophagy induction.
The dispersion journey of LC3 between cytoplasm and nucleus had many postulations and hypothesis. Huang and Liu (19), Nowak et al. ${ }^{(21)}$, and Murrow and Debnath ${ }^{(22)}$ suggested that under full basal conditions, siturin1 (SIRT1) activity is low and LC3 is acetylated and localized in the nucleus in an inactive form. Upon stressful conditions, activated SIRT1 deacetylates LC3 that allows interacts with the delta-opioid receptor (DOR), and then both proteins relocate from the nucleus to autophagosomes in the cytoplasm. They added that deacetylated LC3 interacts with Atg7 and other components of the ubiquitin-like conjugation machinery, leading to LC3 conjugation onto phosphatidylethanolamine (PE) and its incorporation into the early autophagosomal membrane.

Recent studies have highlighted the importance of nuclear autophagy in physiological and pathological processes. Nevertheless, the underlying molecular mechanisms remain to be explored ${ }^{(23)}$. Therefore, further studies with a larger sample size could ensure the role of LC3 nucleophagy in psoriasis.

Therefore, in this study, we could assume that nucleocytoplasmic localization of LC3 may be responsible for exaggerated autophagy induction. This could be a new insight into the target therapies against psoriasis. However, further studies about the exact role of LC3 in psoriasis are recommended.

\section{REFERENCES}

1. Sil P, Wong S, Martinez J (2018): More than skin deep: autophagy is vital for skin barrier function. Frontiers in Immunology, 9: 1376-82.

2. Sharaf A, Ibrahim A (2017): Quality of life of patients with Psoriasis in Alexandria- Egypt. IOSR Journal of Nursing and Health Science, 6(1): 17-29.

3. Al-Dhubaibi M (2018): Association between Vitamin D deficiency and psoriasis: An exploratory study. Int $\mathbf{J}$ Health Sci., 12(1): 33-39.

4. Nagar R (2017): Autophagy: A brief overview from the perspective of dermatology. Indian J Dermatol Venereol Leprol., 83(3): 290-297.

5. Cui D, Huo S, Wang $X$ et al. (2020): Establishment of canine macrophages stably expressing GFP-tagged canine LC3 protein for effectively detecting autophagy. Molecular and Cellular Probes, 49: 101-3.

6. Li X, He S, Ma B (2020): Autophagy and autophagyrelated proteins in cancer. Molecular Cancer, 19(1): 1216.

7. Louden B, Pearce D, Lang W et al. (2004): A Simplified Psoriasis Area Severity Index (SPASI) for rating psoriasis severity in clinic patients. Dermatology Online Journal, 10(2): 7-9.

8. Chularojanamontri L, Kulthanan K, Suthipinittharm P et al. (2015): Clinical differences between early- and late- onset psoriasis in $\mathrm{T}$ hai patients. International Journal of Dermatology, 54(3): 290-294. 
9. Alshenawy H, Hasby E (2011): Immunophenotyping of dendritic cells in lesional, perilesional and distant skin of chronic plaque psoriasis. Cellular Immunology, 269(2): 115-119.

10. Komine M, Karakawa M, Takekoshi T et al. (2007): Early Inflammatory Changes in the Perilesional Skin of Psoriatic Plaques: Is there Interaction between Dendritic Cells and Keratinocytes?. Journal of Investigative Dermatology, 127(8): 1915-1922.

11. Yue L, Ailin W, Jinwei $Z$ et al. (2019): PSORI-CM02 ameliorates psoriasis in vivo and in vitro by inducing autophagy via inhibition of the PI3K/Akt/mTOR pathway. Phytomedicine, 64: 153-58.

12. Rendon A, Schäkel K (2019): Psoriasis pathogenesis and treatment. International Journal of Molecular Sciences, 20(6): 1475-79.

13. Guo Y, Zhang X, Wu T et al. (2019): Autophagy in skin diseases. Dermatology, 235(5): 380-389.

14. Samaka R, Basha M, Mansour E (2020): Dose the autophagy-related gene7 (ATG7) have a role in nonmelanoma skin cancer?. Clinical, Cosmetic, and Investigational Dermatology, 13: 49-58.

15. Wang $Z$, Zhou $H$, Zheng $H$ et al. (2020): Autophagybased unconventional secretion of HMGB1 by keratinocytes plays a pivotal role in psoriatic skin inflammation. Autophagy, 16: 1-24.
16. Fu N, Yang $X$, Chen $L$ (2018): Nucleophagy Plays a Major Role in Human Diseases. Current Drug Targets, 19(15): 1767-1773.

17. Kraft L, Manral P, Dowler J et al. (2016): Nuclear LC3 associates with slowly diffusing complexes that survey the nucleolus. Traffic, 17(4): 369-399.

18. Shim M, Nettesheim A, Hirt J et al. (2020): The autophagic protein LC3 translocates to the nucleus and localizes in the nucleolus associated with NUFIP1 in response to cyclic mechanical stress. Autophagy, 16(7): 1248-1261.

19. Huang R, Liu W (2015): Identifying an essential role of nuclear LC3 for autophagy. Autophagy, 11(5): 852-853.

20. Huang R, Xu Y, Wan W et al. (2015): Deacetylation of nuclear LC3 drives autophagy initiation under starvation. Molecular cell, 57(3): 456-466.

21. Nowak J, Archange $\mathrm{C}$, Tardivel-Lacombe $\mathrm{J}$ et al. (2009): The TP53INP2 protein is required for autophagy in mammalian cells. Mol Biol Cell, 20(3): 870-881.

22. Murrow L, Debnath J (2015): A Nuclear Option That Initiates Autophagy. Molecular Cell, 57(5): 393-395.

23. Luo M, Zhao X, Song $Y$ et al. (2016): Nuclear autophagy: An evolutionarily conserved mechanism of nuclear degradation in the cytoplasm. Autophagy, 12(11): 1973-1983. 